\title{
Holt-Oram Syndrome Revisited. Two Patients in the Same Family
}

\author{
José Dario Frota Filho, Wagner Pereira, Tiago Luiz Luz Leiria, Mario Vallenas, Paulo E. Leães, \\ Celso Blacher, Eraldo Lúcio, Fernando A. Lucchese
}

Porto Alegre, RS - Brazil

\begin{abstract}
Holt-Oram syndrome was first described in 1960 as an association of familial heart disease and musculoskeletal abnormalities. The most important findings include atrial septal defects, atrioventricular conduction abnormalities, vascular hypoplasia, and upper limb musculoskeletal deformities. We report two patients with this syndrome in the same family and discuss the variability of the musculoskeletal abnormalities and their association with the cardiac morphologic defects. Both patients in this study had associated eosinophilia, which has not been reported in the literature.
\end{abstract}

In 1960, Mary Holt and Samuel Oram ${ }^{1}$ described this syndrome for the first time and, since then, different authors have reported on approximately 200 patients. The presence of both cardiac and musculoskeletal abnormalities has inspired a series of names for this syndrome, such as "heart-hand syndrome", "heart upper-limb syndrome" and "upper-limb cardiovascular syndrome", among others. McKusick ${ }^{2}$ created the name Holt-Oram syndrome, when describing a case in which a mother and her daughter were affected.

Holt-Oram syndrome is an autosomal dominant disorder, caused by mutations on chromosome $12 \mathrm{q} 24.1^{3}$ that inactivate the TBX5 gene. The criteria for diagnosing this syndrome include abnormalities of the thumb (triphalangia, hypoplasia or aplasia) and congenital heart disease. The most frequent cardiac abnormalities are atrial septal defects and arrhythmia, both found in the 2 patients studied.

Patient 1 - A 23-year-old male patient, with a height of $166 \mathrm{~cm}$ and weight of $50 \mathrm{~kg}$, presented with palpitations and syncope. He had bilateral agenesis of the first finger and of the thenar area, bilateral absence of the thenar fold, pronation and supination movements absent on the right side and limited on the left side, restricted active mobility of the wrists during flexion, and pectus excavatum (fig. 1). His heart auscultation showed fixed splitting of the second

Hospital São Francisco da Santa Casa de Porto Alegre

Mailing address: José Dario Frota F ${ }^{\circ}$ - Rua Freitas Cabral 305/602 - 90690-130 Porto Alegre, RS, Brazil heart sound (S2) and a systolic heart murmur 4+/6+, with its epicenter in the pulmonary region. The electrocardiogram revealed an ectopic atrial rhythm, incomplete block of the right branch of the His bundle, and an enlarged right ventricle. The echocardiography revealed a large atrial septal defect of the ostium secundum type of hemodynamic importance, enlarged right chambers, pulmonary arterial hypertension (systolic pressure: $59 \mathrm{mmHg}$ ) and slight tricuspid regurgitation. Eosinophilia of $31 \%$ was present. The findings described were confirmed during surgery.

Patient 2-A 19-year-old male patient, with a height of $165 \mathrm{~cm}$ and weight of $50 \mathrm{~kg}$, presented with palpitations and syncope. In the previous months, he had complained of accelerated heartbeat followed by a slow heart rhythm (according to the patient's report) with feelings of imminent death occurring at least twice a week. He had bilateral dysgenesis of the forearms and hands, absence of interphalangeal and thenar cutaneous folds, absence of the thumbs, laterally rotated and abducted hands, bilateral complete limitation of pronation and supination movements, deviation of the axis of the wrists with restricted adduction and abduction, atrophy of the flexor muscles of the fingers, and pectus excavatum (fig. 2). His heart auscultation showed an irregular heart rate of approximately $43 \mathrm{bpm}$, a loud second heart sound without splitting, and a systolic heart murmur $6+/ 6+$, which could be heard throughout the precordial area and whose epicenter was situated in the pulmonary region. The radial and ulnar pulses could not be palpated, but the perfusion of the upper limbs was adequate. Electrocardiography showed ectopic atrial rhythm, bouts of ventricular premature beats, second degree atrioventricular block of the Mobitz I type and incomplete right branch block of the His bundle. Echocardiography showed a large atrial septal defect of the ostium secundum type of hemodynamic importance, enlarged right chambers, pulmonary arterial hypertension and dilation of the coronary sinus, due to a probable drainage of the left superior vena cava. The eosinophil count was $31 \%$. The findings described were confirmed during surgery.

Family background and genealogy - The pedigree diagram (fig. 3) shows a nonconsanguineous couple (I) with 


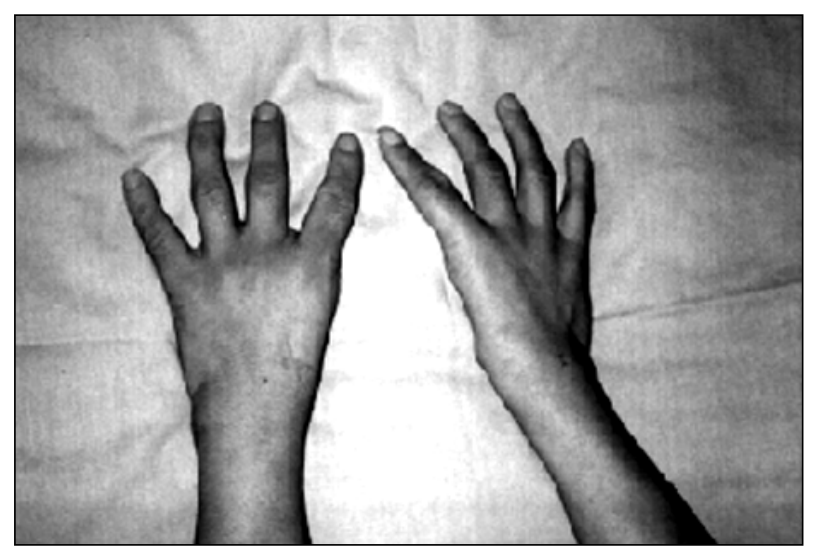

Fig. 1- Note the absence of thumbs.

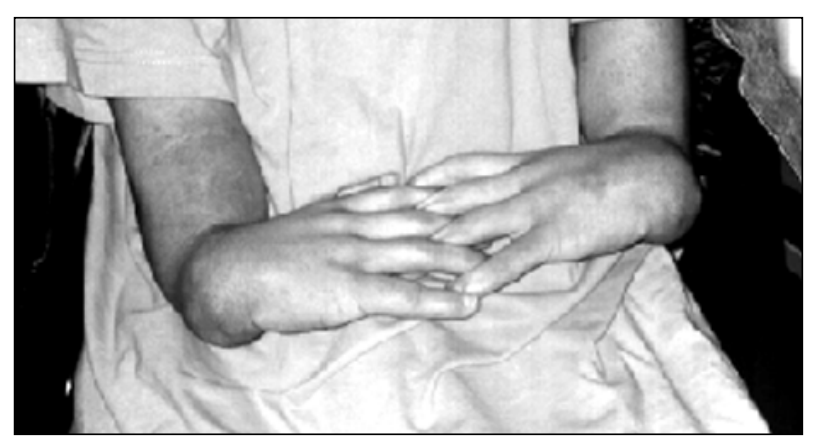

Fig. 2-Radio-humeral hypoplasia and absence of thumbs.

5 children (II), 3 males and 2 females. The father, already deceased, had undergone heart surgery several years earlier, but the hospital records and the death certificate did not define the heart disease or the cause of death. One of the patient's brothers had congenital heart disease (according to the patient's report) and had died suddenly, but due to lack of available information in official registries, it was not possible to reach a conclusion regarding either the etiology of the disease or the cause of death. Another sister of this patient is reported to have limited wrist movements. In addition, one of the brothers married a nonconsanguineous woman, and this couple had two daughters, of whom one has thumb abnormalities (according to the patient's report). None of the other components of the genealogy has been examined so far; therefore, confirmation of other complementary elements of the syndrome are lacking. In the pedigree diagram exhibited in figure 3, the patients with Holt-Oram syndrome are represented by black squares and the patients probably affected are represented by black circles.

Surgical procedure-Both patients were operated upon on the same date. The surgery consisted of atrioseptoplasty with insertion of a bovine pericardial graft during routine extracorporeal circulation. The postoperative period was uneventful. In patient 2 , a definitive dual-chamber pacemaker (DDD) was inserted before hospital discharge due to previous symptoms and to electrocardiographic abnormalities. Patient 1 will be followed up on an outpatient care basis to evaluate the need for definitive pacing in the future.

\section{Discussion}

Starke et al ${ }^{4}$ and Gall et al ${ }^{5}$ published some reports on Holt-Oram syndrome occurring in families, but the literature also provides isolated case reports ${ }^{6}$. More recently, the studies have been focusing on the genetic study of this condition, associating the morphological abnormalities with a mutation on an autosomal dominant gene (TBX5), which resides on the long arm of the chromosome $12 \mathrm{q} 24.1^{3}$. Until now, the correlation between the severity of the upper limb abnormalities and the cardiac abnormalities could not be established ${ }^{7}$.

The most frequent cardiac abnormalities are atrial septal defects and arrhythmias ${ }^{8}$ and, among the former, atrial septal defects of the ostium secundum type occur in $34 \%$ of the patients ${ }^{9}$. In patient 2 , the surgical findings included an atrial septal defect whose greatest orthogonal axes measured approximately $6 x 4 \mathrm{~cm}$, a finding that may result in practical absence of atrial septum. Bernard et al ${ }^{10}$ also described this abnormality. The literature also reports cardiac conduction defects, such as those described in our 2 patients ${ }^{7}$. Patient 2 showed drainage of the left superior vena cava into the coronary sinus, which was confirmed during the surgical procedure. Pulmonary arterial hypertension may occur in a significant number of patients and is generally a result of the excessive pulmonary flow caused by the atrial septal defect, as shown in both patients. However, this hypertension may also occur as primary pulmonary arterial hypertension ${ }^{11}$.

Skeletal abnormalities spare the lower limbs. This occurs because the mutant gene interferes with the embryonic differentiation during the $4^{\text {th }}$ and $5^{\text {th }}$ weeks of pregnancy, when the lower limbs are not yet differentiated ${ }^{12}$. The upper limb abnormalities are located predominantly at the radial margin and occur as triphalangia, hypoplasia or absence of thumbs (as occurred in the 2 patients reported) (fig. 3), or, additionally, as thumbs located at the same level (both patients); short clavicle; abnormalities of the $5^{\text {th }}$ finger and abnormalities of the scapula (patient 2); pectus excavatum

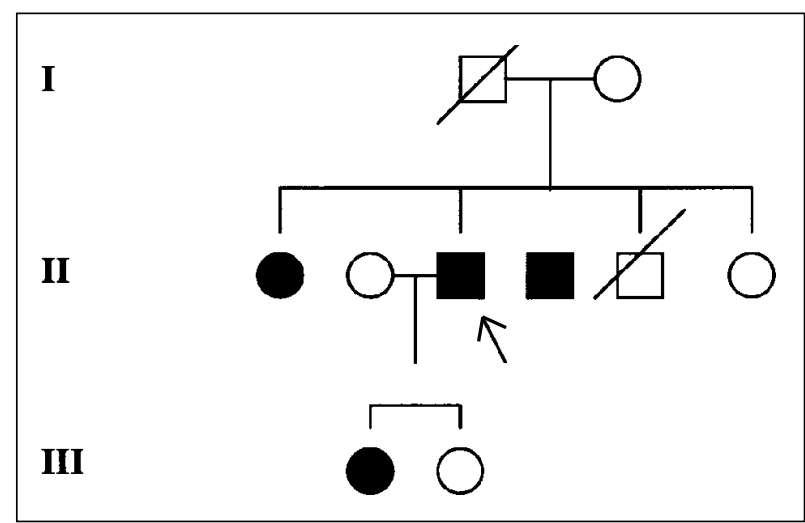

Fig. 3 - Pedigree diagram. 
(both patients); humeral abnormalities (patient 2); ulnar abnormalities, radioulnar synostosis and prominent medial epicondyle. Impaired mobility of the upper limbs may be encountered regardless of the skeletal abnormalities ${ }^{7}$. In patient 1 , a slight bilateral muscular hypotrophy of the forearms and hands was noted, involving mainly the flexor muscles, with hypertrophy of the abductor and adductor muscles of the hands. Pronation and supination movements at the right side were absent and the active movements of the wrists were severely restricted, mainly during flexion. In patient 2, bilateral limitation of the pronation and supination movements occurred, with deviation of the axis of the wrists and limited abduction and adduction. In spite of the abnormalities described, the hands of this patient performed fairly.

The last topic that deserves mention is the eosinophilia found in both patients, in whom no known cause for this abnormality could be found. In some other genetic disorders, eosinophilia of unknown cause occurs in $63 \%$ of the patients, as observed in thrombocytopenia and absence of the radius (TAR) ${ }^{7}$. We have not found any references in the literature to the association between eosinophilia of unknown origin and Holt-Oram syndrome, and this raises the possibility that, in our patients, this was an incidental finding or a feature of this syndrome not yet described.

\section{References}

1. Holt M, Oram S. Familiar heart disease with skeletal malformation. Br Heart J 1960; 22: 236: 42 .

2. McKusick VA. Medical genetics. J Chron Dis 1961: 14-1.

3. Basson CT, et al. Mutations in humans cause limb and cardiac malformations in Holt-Oram syndrome. Am J Med Genet 1996; 65: 128-32.

4. Starke H, Schimke RN, Dunn M. Upper limb cardiovascular syndrome. A family study. Am J Cardiol 1967; 19: 588.

5. Gall Jr JC, Stern AM, Cohen MN, et al. Holt-Oram syndrome: clinical and genetic study of a large family. Am J Hum Genet 1966; 18: 187

6. Donadeo V, Solarino M, Alfieri O, et al. Holt-Oram syndrome: cardiologic, radiologic and genetic evaluation. Rev Port Cardiol 1995; 14: 265-6.

7. Jones KL. Holt-Oram Syndrome. In: Smith's Recognizable Patterns of Human Malformations. $5^{\text {th }}$ ed. Philadelphia: WB Saunders Co., 1997: 316-7.
8. Shashidhar Pai G. Heart-Hand Syndrome. In: Birth Defects Encyclopedia, Mary Louise Buyse. London: Blackwell Scientific Publications Inc., 1990: 850-2.

9. Newbury E, Leanage R, Reaburn JA, et al. Holt-Oram syndrome: a clinical genetic study. Genet Couns 1996; 7: 323-4.

10. Bernard J, Tessier JP, DiardF, et al. Association of major malformation of the upper limbs (phocomielia) and congenital cardiopathy: extension of Holt-Oram syndrome. Ann Radiol 1972; 15: 901.

11. Kuhn E, Shaaf J, Wagner A. Primary pulmonary hypertension, congenital heart disease and skeletal abnormalities in three gengerations. Jap Heart J 1963; 4: 205.

12. Araújo Filho R, Alves PM, Carakushansky G, et al. Síndrome de Holt-Oram com persistência de canal AV comum, forma total. Relato de um caso. Arq Bras Cardiol 1983; 40: 397-402. 